\title{
Response to the Coronavirus Disease-2019 Pandemic: Lessons Learned from the Taiwan Model
}

\author{
Charles $\mathrm{Wu}^{1}$, Catherine $\mathrm{Wu}^{1} \&$ Kun Chan $\mathrm{Wu}^{2}$ \\ ${ }^{1}$ Morrison Academy, Taichung, Taiwan \\ ${ }^{2}$ Wu Kun Chan Clinic, Miaoli, Taiwan \\ Correspondence: Kun Chan Wu, Wu Kun Chan Clinic, Miaoli, Taiwan. E-mail: \\ wupedhanwupedhan@gmail.com
}

Received: August 5, 2020

Accepted: September 9, 2020

Online Published: September 24, 2020

doi:10.5539/ass.v16n10p16

URL: https://doi.org/10.5539/ass.v16n10p16

\begin{abstract}
The severe acute respiratory syndrome coronavirus 2 (SARS-CoV-2), or coronavirus disease-2019 (COVID-19), emerged in December 2019 in Wuhan, China and has since then spurred a global pandemic (Lai et al., 2020). Taiwan and China, separated only by $130 \mathrm{~km}$ across the Taiwan Strait, have frequent cross-strait interactions with each other; millions of people travel to and from between the two countries (Wang \& Lin, 2020). Considering these facts, Lauren Gardner, an associate professor at the Johns Hopkins University, even predicted that Taiwan will have the second highest number of COVID-19 cases among the world using a metapopulation model (Gardner et al., 2020). However, with a population of 23.7 million people, Taiwan leads one of the least COVID-19 cases worldwide. With the help of technology, swift reactions, advanced deployment of resources, and complete transparency, the Taiwan model has made its success. By analyzing the actions taken and how they functioned in Taiwan in preventing a nationwide epidemic, other countries may benefit in understanding how to design better models for the prevention of future epidemics and pandemics.
\end{abstract}

Keywords: COVID-19, pandemic, SARS-CoV-2, Taiwan model

\section{Introduction}

The coronavirus disease-2019 (COVID-19) outbreak was first reported in Wuhan, Hubei Province, China in December 2019 and has spread across China (World Health Organization a, 2020). However, in the next few months, this epidemic did not seem to arouse a global health concern (Lai et al., 2020). Nevertheless, the virus spread to the world like wildfire. By July 30, 2020, the World Health Organization (WHO) reported more than 16523815 confirmed cases in 216 countries/regions and 655112 confirmed deaths (World Health Organization b, 2020).

The first confirmed case of COVID-19 in Taiwan - a neighbor to China with recurrent long-term, close, cross-strait relationships - was from its overseas counterpart. The disease entered the island on January 21, 2020; the victim was a 55-year-old Taiwanese businesswoman who returned to Taiwan from Wuhan (Ministry of Health and Welfare, 2019a). This event marks the onset of the COVID-19 outbreak in Taiwan.

The epidemic soon reached its peak within two months. However, through the implementation of aggressive policies under the Infectious Control Act and the reactivation of the Central Epidemic Command Center (CECC), the severe acute respiratory syndrome coronavirus 2 (SARS-CoV-2) epidemic declined rapidly after April (Centers for Disease Control, 2018). As of July 30, 2020, Taiwan has only 467 confirmed cases and seven deaths for COVID-19, and the case numbers resulting from the community spread of the disease remained zero for months (Centers for Disease Control, 2018). Taiwan's model for combating COVID-19 has evidently been a success. Following its success, Taiwan has the chance to turn its attention to economic stimulation. This review study presents the timeline, methodology, and institutions that took part in stimulating Taiwan's success in combating SARS-CoV-2.

\section{Methods}

The content of this paper was mainly gathered from personal observations when attending conferences, seminars, and video meetings on COVID-19; from clinicians in the Hospital Authority; and from the information released from the CECC in Taiwan. Other sources were from press reports, personal interviews, and websites on 
COVID-19 provided by the health authorities, the US Centers for Disease Control and Prevention, the WHO, and the Centers for Disease Control (CDC) in Taiwan.

\section{What Is the Taiwan Model for Combating COVID-19? What did Taiwan Meet, Think, and React to This Outbreak?}

The severe acute respiratory syndrome (SARS) outbreak in 2003, a coronavirus-induced epidemic, killed 73 people in Taiwan (Wang et al., 2020). SARS may have taken many lives and hurt the economy, but it more importantly served as a caveat for enhancing disease prevention strategies. In the wake of the SARS epidemic, Taiwan set up its National Health Command Center (NHCC), a unified disaster management center that "includes the CECC, the Biological Pathogen Disaster Command Center, the Counter-Bioterrorism Command Center, and the Central Medical Emergency Operations Center" (Taiwan Centers for Disease Control, 2018). The NHCC essentially coordinates with government agencies and organizations in performing epidemic prevention work by facilitating different departments and commanding different actions to be taken, altogether harmoniously utilizing available resources and achieving its goal of disease prevention (Department of Disease Control, 2020).

Taiwan also rebuilt many standard operating procedures (SOPs) for epidemic prevention and promulgated a detailed Infectious Disease Control Act after the SARS epidemic. The act allows the Ministry of Health and Welfare (MOHW) to partition all necessary labor, funding, and capital toward disease control from the Executive Yuan, an executive branch of the Taiwanese government. It also allows for the establishment of the CECC, a temporary agency consisting of a congregation of decision-makers who will determine operations during an epidemic (Taiwan Centers for Disease Control, 2018a). With this act and the NHCC, the government now has the power to designate medical institutions as emergency, patient-isolating hospitals during the outbreak and implement measures to avoid in-hospital infections. Standard procedures for the monitoring and notification of infectious diseases have also been developed, and border quarantine procedures have been optimized. The government also formalized compulsory home quarantine for disease-infected people or people who may have come in contact with an infected person(s) from all affected areas and requested that the relevant authorities ensure adequate emergency medical supplies at all times (Taiwan Centers for Disease Control, 2018a).

Another distinguishing feature about Taiwan's anti-epidemic prowess is its health insurance system. In 20162017, the National Health Insurance covers $99.6 \%$ of the population, creating universal healthcare and making visits to a healthcare professional efficient, undaunting, and affordable (Lee et al., 2016). Thus, if people suspect themselves of having COVID-19, they need not worry that they cannot afford to get tested. As such, Taiwan's health insurance system also took a pivotal stand in Taiwan's success of COVID-19 containment.

\subsection{Remaining Vigilant and Proactive in Recognizing the Crisis}

After the overwhelming experience of the SARS outbreak, officials in Taiwan became especially keen in following news regarding epidemics around the world, especially its neighboring regions. Taiwan's vigilance has proven to work in its favor in the earlier stages of the COVID-19 pandemic. In the early morning on December 31, 2020, Luo Yi-jun, the deputy director of Taiwan's CDC, found information from the social media platform "PTT" stating that, "There were 27 cases of pneumonia been found recently in Wuhan, China, and 7 cases are critically ill in isolation therapy. 2 cases improved" (Borak, 2020). Reacting to this unsuspecting warning, Luo confirmed the coming of an epidemic the following morning and notified the WHO International Health Regulations window, which regulates and monitors global health issues (Ministry of Health and Welfare, 2019b). On that same day, China notified the WHO of its atypical pneumonia cases in Hubei. The Taiwanese government reacted in cautious alacrity by immediately imposing heightened disease screening for immigrants arriving in Taiwan from Hubei Province, China - where Wuhan is located - along with travel warnings on Hubei (Ministry of Health and Welfare, 2019; Jiang, 2020). Since then, Taiwan took hundreds of proactive action items to distance itself from a major outbreak (Wang et al., 2020).

Among these actions would be dispatching a team of epidemiologists and scientists on January 12, 2020 to mainland China in order to gather more intel on this enigma of a disease. The experts sent on the expedition sensed an ominous outlook of the disease (Ministry of Health and Welfare, 2020b). In response to this grave situation, Taiwan's CDC said on January 2, 2020, "People returning from Wuhan within 14 days, if they have fever and respiratory symptoms, they should seek medical treatment as soon as possible, and the doctor will transfer the patient and notify another doctor responsible for COVID-19 testing" (Ministry of Health and Welfare, 2019a). What this means is that passengers returning from Hubei who display such symptoms had to be quarantined at home or even in hospitals if a patient requires medical attention. Hospitals were also assigned for mandatory COVID-19 testing and documenting for the CECC (Ministry of Health and Welfare, 2020a; Wang et 
al., 2020). These preventive actions were all taken prior to Taiwan's first case on January 21 . The remaining portion of these sections will shed more light on the specific actions of the Taiwan model.

\subsection{Setting Up a Command Center (CECC) via the Laws and Undertaking Swift Reactions}

On January 15, 2020, Taiwan declared SARS-CoV-2 as a Class 5 Infectious Disease (Ministry of Health and Welfare, 2019a). Five days later, the Taiwan CDC officially activated the CECC under the Infection Control Act and the NHCC for dealing with SARS-CoV-2 cases (Ministry of Health and Welfare, 2019a). The minister of the MOHW, Chen Shih-chung, was assigned an additional job as commander of the CECC. Under Chen, the CECC rapidly "coordinated efforts by various ministries, including the ministries of transportation, economics, labor, and education and the Environmental Protection Administration, among others, in a comprehensive effort to counteract the emerging public health crisis" (Wang et al., 2020). Five weeks later, the CECC had created 124 action items to strengthen its defense against the virus (Wang et al., 2020; Ministry of Foreign Affairs, 2020). The CECC's competence in efficiently creating solutions to problems - it was able to come up with at least three action items per day - has been one of the forefronts to Taiwan's success.

\subsection{Implementing Rapid Border Control Measures}

Taiwan took many tough actions early on in the epidemic. While many actions aimed at domestic disease prevention, Taiwan gradually adjusted the immigration regulations to foreign intrusion of COVID-19. On January 26, 2020, about two weeks after Taiwan declared the virus as a Class 5 Infectious Disease, Taiwan restricted passenger arrivals from Wuhan (Ministry of Health and Welfare, 2019a). Taiwan was the first to do so in the world. In the next couple of weeks, Taiwan expounded on the travel restriction by banning almost all flights arriving from cities in China (Ministry of Health and Welfare, 2019a). More changes were made in the following months, but the chief idea is that Taiwan kept its travel bans while adjusting its extent according to the condition of global epidemics. Taiwan recognized how the virus was permeating through the world, and on March 19, 2020, all immigrants except personnel holding residence/special permits were banned from entering Taiwan (Ministry of Health and Welfare, 2020d). Prior to March 19, 2020, immigrants entering Taiwan from high-risk areas were quarantined for 14 days; with the spread of the virus internationally, all immigrants were required to be quarantined for 14 days.

\subsection{Continuing Active Case Documentation and Containment}

\subsubsection{Using Technology to Detect and Track Cases}

Following the 2003 SARS outbreak, Taiwanese authorities issued temperature-screening monitors in airports to detect and document immigrants with fevers, which is a symptom of the coronavirus (Taoyuan International Airport Co., Ltd., 2020). Conveniently, temperature monitors have assisted Taiwan in catching potentially sick patients of COVID-19. Temperature monitors thus served as a first line of defense against the virus.

Taiwan also used its existing National Health Insurance (NHI) system to its advantage. Specifically, the National Health Insurance Administration (NHIA) coordinated with the National Immigration Agency to incorporate individual's past 14-day travel history into their NHI cards. Hence, medical institutions can retrieve information from the "Hubei Province Tourism History" through the health insurance medical information cloud query system. This mission was accomplished in only one day. The system was modified later on February 18 when patient travel histories abroad were made available to all medical facilities (i.e., hospitals and clinics) in Taiwan by reading the data recorded in the NHI identification cards (Wang et al., 2020).

Another example of the implementation of technology is the Entry Quarantine System (EQS). It is a program launched on February 14 for travelers to complete a mandatory health declaration form before emigrating or immigrating into the country. The data collected were then sent directly to Taiwan's CDC (Wang et al., 2020). With the institution of the EQS, medical staff can retrieve information on individual's past 14-day overseas travel history through the "MediCloud" medical information query system. Then, medical personnel can more easily determine the measures that need to be taken by weighing a patient's symptoms in conjunction with their travel history (Ministry of Health and Welfare, 2020c; Wang et al., 2020). The genius of the EQS lies in its efficiency as data can be easily collected all by scanning a QR code and completing a form.

As the pandemic worsened, all the people coming from abroad, not only those from Hubei, were placed under mandatory 14-day home quarantine. To cope with and facilitate the large number of people in quarantine, Taiwan also implemented a technology to assist it. One such innovation is that immigrant information is integrated into the 14-day home care "anti-epidemic tracking system" and the tracking alarm "electronic fence system" in order to accurately grasp the whereabouts of relevant personnel, which identified anyone who did not follow the quarantine protocols and advisories (i.e. staying within the household). Moreover, once a suspicious case appears, 
GPS can allow contact tracing or track people who came in contact with others who tested positive for COVID-19 or people who are reported as a suspected case; once found, these people will also be confined to a quarantine period. Consequences for a person leaving their household during quarantine may include heavy fines (Ministry of Health and Welfare, 2020c).

\subsubsection{COVID-19 Testing Policies in Taiwan}

By establishing a national inspection network and building a community acquisition and inspection network and a precise inspection strategy, the CECC was able to gradually increase Taiwan's overall testing volume. At present, there are 52 designated inspection institutions and 161 designated community collection institutes. The maximum daily capacity of SARS-CoV-2 RT-PCR test has exceeded 6,474 RT-PCRs, and the test results can be obtained within 4 to $6 \mathrm{~h}$ (Department of Disease Control, 2020). To say the very least, COVID-19 testing in Taiwan is efficient and accessible.

If a patient has a fever or has history of travel, occupation, contact, or cluster (TOCC), either revealed by patient's testimonies or by the data retrieved from his/her NHI identification card indicating a possible case of COVID-19, he/she will be escorted to the said community collection institutes for SARS-CoV-2 infection testing (Department of Disease Control, 2020).

The 1922 "Communicable Disease Reporting and Consultation Hotline" was also made available by the CECC to allow citizens to seek further health information or to notify officials on suspicious symptoms or cases of COVID-19 in people around them. It has been available 24/7 all year round and is toll free (Ministry of Health and Welfare, 2020e).

At the appointed institutes, testing for SARS-COV-2 is completely free. Patients who are tested positive for the SARS-CoV-2 infection will receive isolation treatment and observation in negative-pressure rooms in hospitals (Department of Disease Control, 2020). However, Taiwan's government does not neglect its patients, but ensures that governmental funds are used for caring its people. "If patients were forced to be isolated, during this period, the government would pay for the food, lodging and medical care. So almost no one would avoid seeing the doctor because they can't pay for health care" said Yotaka (2020), as cited in Sui (2020). After isolation therapy, patients can be discharged only when they have negative RT-PCR results of SARS-CoV-2 for three times.

To ensure that there are not any loose cases, authorities also track down suspected associates of the confirmed cases. Taking extreme precaution, the authorities would then place anyone who came in contact with the patient in quarantine as well (Wang et al., 2020). In summary, Taiwan's COVID-19 testing is not only comprehensive, but empathetic toward its people.

\subsection{Maintained Assertive Resource Allocation to Ensure the Availability of Supplies}

More and more evidence points toward how wearing masks "reduces the transmissibility per contact by reducing transmission of infected droplets in both laboratory and clinical contexts" (Howard et al., 2020). If people wear masks as a collective, experts predict that COVID-19 cases will take a sharp decline. Despite these facts, the wearing of masks has incited fierce disputes in the Western society at the beginning of this pandemic. However, based on the traditional habit and advanced experience from the SARS epidemic, the government and people in Taiwan quickly understood the importance of mask-wearing without doubts and were willing to put masks on properly (i.e., covering the mouth and over the nostrils) immediately at the beginning of this pandemic. People in Taiwan saw masks as an important apparatus and crucial epidemic protection action, which made them feel safe and therefore not as panicked and anxious, contrary to the social atmosphere in the rest of the world at the time. However, one problem still remained: even though everyone was willing to wear masks, will there be enough masks for everyone? In other words, given that the citizens and, more importantly, medical personnel needed and tended to stock masks for themselves, how did the government generate sufficient supplies of masks and medical resources?

\subsubsection{Managing Inventories and Dispatching Epidemic Prevention Materials}

To ensure that resources are not monopolized by a few firms and are distributed in a way that allows for access of imperative disease-combatting resources for all peoples, the government collected the product lists and license information of medical equipment needed for epidemic prevention from companies and actively investigated the domestic supply and demand sales (Ministry of Health and Welfare, 2020f). Taiwan also kept a close eye on the daily production and distribution of domestic medicinal alcohol and coordinated with Taiwan Tobacco and Liquor Corporation and Taiwan Sugar Corporation to increase the production of medicinal alcohol for epidemic prevention in order to meet the needs of medical facilities and daily consumption from the public (Ministry of Health and Welfare, 2020f). 
The CECC also banned all exports of masks on January 24, 2020 to safeguard an adequate supply of masks for Taiwan. Moreover, from January 31, 2020, all general and surgical masks produced in Taiwan are to be requisitioned and distributed by the command center. After the requisition of masks, local governments could uniformly distribute epidemic prevention materials to medical care units, civil affairs, fire departments, police departments, and other epidemic prevention staff within their jurisdictions to ensure supply efficiency and scheduling flexibility. The CECC allocated its resources efficiently with priority to medical institutions for use by coordinating and matching epidemic prevention materials such as forehead guns, masks, isolation gowns, medicinal alcohol, etc. All these actions ensured that Taiwan would maximize its epidemic prevention materials and minimize unsteady changes from capricious manufacturers by facilitating all productions (Ministry of Health and Welfare, 2020f).

Moreover, the CECC "set up new production lines and dispatched soldiers to staff factories, significantly increasing production" (Sui, 2020). At the end of January 2020, Taiwan's daily mask production capacity was only 1.88 million pieces. By March 20, Taiwan had completed the construction of 92 additional mask production lines, including two additional production lines for surgical bandage masks and 90 conventional mask production lines. The overall project took 40 days, and increased the daily production capacity to 15 million pieces (C. J. Wang et al., 2020). Each mask was sold at 16 cents USD, making them affordable to all.

Knowing that Taiwan has a high density of convenience stores and pharmacies and to ensure everybody has enough masks to wear, the CECC delivered the masks to the appointed "The Four Main chained Convenience stores" - 7-Eleven, FamilyMart, Hi-Life, and OK supermarkets - and pharmacies. Then, judging by the national stock of masks, the CECC adjusted the availability of masks, or the number of masks in circulation, for people to buy. Since February 6, 2020, the CECC announced that everyone has a quota to buy two masks per week. By April 9, 2020, that quota was adjusted to nine masks for adults and 10 masks for children every 14 days. To keep mask distribution fair and organized and to prevent people from purchasing over their quota, everyone's mask-buying records are documented on their NHI identification card. This way, personnel of pharmacies and convenience stores can obtain the correct purchasing history of masks. The mask-purchasing process was even more refined and convenient when the real-name mask system 3.0 was introduced on April 22, 2020, which allowed people to pre-purchase and receive masks by simply using their NHI card on a supermarket business machine, which is located in more than 10000 places around Taiwan (Ministry of Health and Welfare, 2020f).

Similar policies are used for other sanitization materials such as alcohol. People and healthcare workers (HCWs) and facilities are allowed to buy alcohol in a regular period of time to ensure fair and enough supplies for everyone.

\subsection{Reassurance and Education of the Public and HCWs}

\subsubsection{Transparency and Trust}

Taiwan's government stands by one rectitude: transparency. Only when information is available to the public would people entrust their lives in the hands of the government and learn medical knowledge and disease prevention techniques that are crucial to stopping the spread of the virus. When there is transparency, the people and the government can stand as one and live through the epidemic with less fear (Sui, 2020). To ensure that information is communicated in that manner, Taiwan employed various actions. Many examples can be presented as follows:

1. The CECC released daily press briefings and held press conferences with Chen Shi-chung (commander of the CECC) and his team that were broadcasted on various television channels to establish transparent and professional media communication with the public. This allowed for an open and mutual understanding of the specific actions taken by the CECC throughout the epidemic. Moreover, by having the commander of the CECC make his appearance to the public on a daily basis, people may feel closer with and trust the authorities more (Ministry of Health and Welfare, 2020e).

2. The CECC also partnered with various media platforms (social media apps, news channels, radio stations, etc.) to broadcast public service announcements on topics such as the correct hand-washing technique, the need for social distancing, the importance of wearing masks, and coughing etiquettes that can limit viral spreads (Sui, 2020). The people that were featured in these media ranged from doctors, scholars, Youtubers, or welcomed and influential celebrities. Infectious or epidemiologist experts were also often invited to attend talk show programs to educate the public (Ministry of Health and Welfare, 2020e).

3. For more efficient spreading of factual epidemic-related data and epidemic investigation results, the government used social media such as the official Line and Facebook to immediately publish the latest 
policies and strategically clarify any rumors on the internet (Ministry of Health and Welfare, 2020e).

4. To ensure that public consultation is available, the CECC expanded the capacity of 1922 anti-epidemic special lines from the original 90 answering lines to 270 lines and increased the relative manpower from the original 16 to 400 customer service staff (Ministry of Health and Welfare, 2020e).

As seen from the examples above, Taiwan utilized its resources to ensure that information is reachable to everyone. As a summary, they did so by directing funds to information spreading on television broadcasts, radio stations, and social media platforms.

\subsubsection{Educated Representatives and Leaders}

Taiwan's frontline HCWs had experienced the era of SARS and were equipped with the SOPs to combat SARS. Thus, the HCWs in Taiwan are used to reading up-to-date literature, journal papers, and articles to educate themselves on health-related information. Because officials keep themselves in the academic loop, they were able to hold frequent meetings and conferences and discuss measures for battling the virus. Again, to maintain transparency, the government also releases new training/meeting information via email, video conferences, etc. from time to time (Wang et al., 2020).

\subsubsection{Guiding Public Facilities}

The CECC established guidelines to instruct SOPs for schools, corporations, restaurants, facilities, and workers on temporary leave. While these guidelines were not strictly enforced, they were available for reference for the public. This gave people such as principals or managers and other leaders in organizations and their people clear ideas on how they can protect themselves and others from COVID-19.

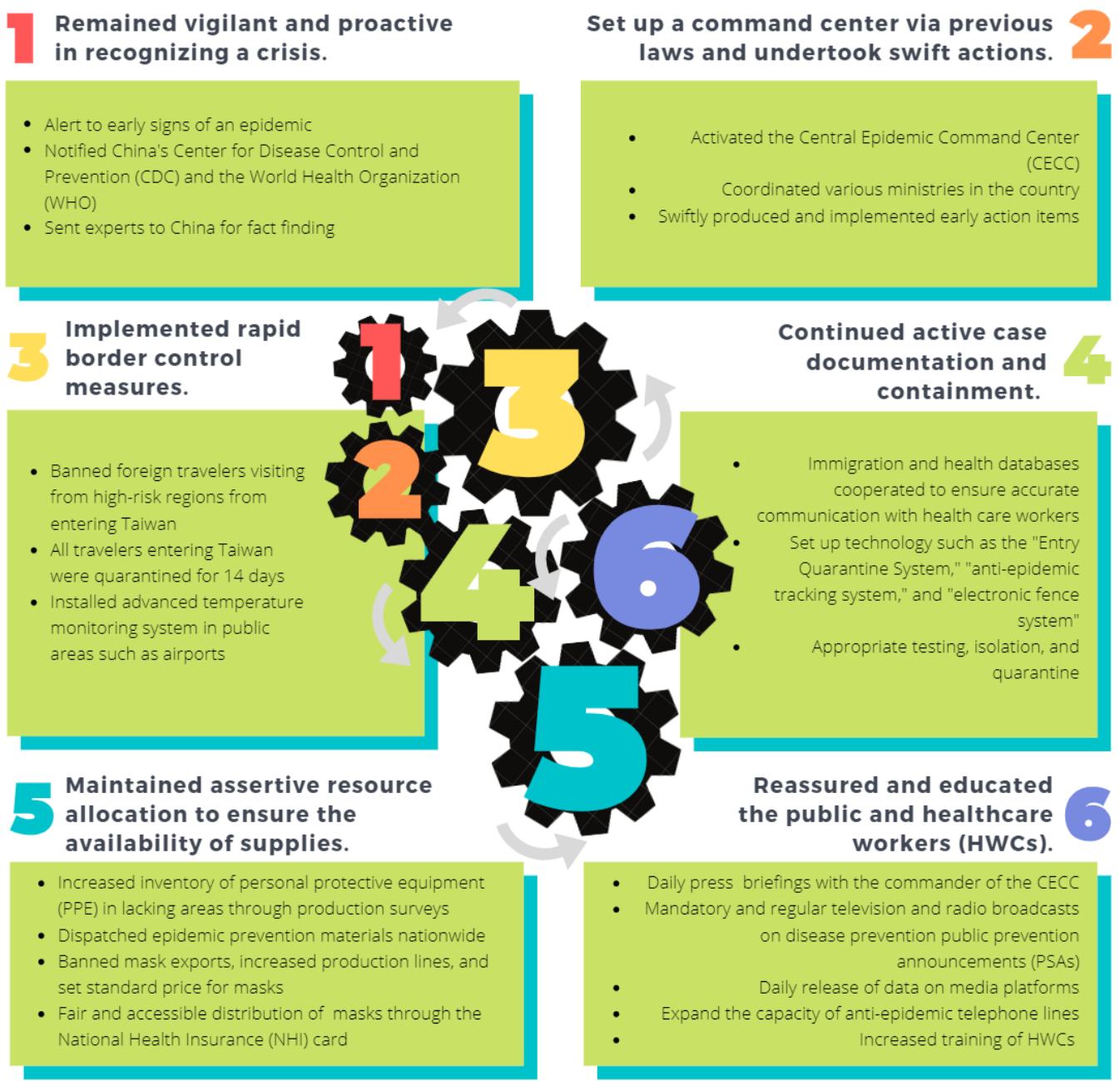

Figure 1. Overlying summary

Taiwan's model for combatting COVID-19 can be summarized into six major actions: remaining vigilant and 
proactive in recognizing the crisis, setting up a command center, implementing rapid border control measures, continuously monitoring active cases, maintaining assertive resource allocation, and educating the public along with HCWs.

\section{What Taiwan Achieved So Far (as of June 30)}

Through swift reactions, the influx of COVID-19 patients had ceased at the border since April 2020 (Centers for Disease Control and Prevention, n.d.). Nearly no cases were missed in Taiwan since the strict border policies were applied and the CECC had worked frivolously in case documentation and tracking suspected individuals (Centers for Disease Control, 2018). Although the first COVID-19 case in Taiwan was confirmed on January 21, 2020 and the epidemic of newly diagnosed cases rapidly reached its height within the subsequent two months, the case numbers were lowered down effectively through the swift policies of containment implemented by the CECC, and Taiwan was ultimately able to "flatten the curve." In other words, no more outbreaks of COVID-19 occurred after April 2020 (Centers for Disease Control and Prevention, n.d.). Since then, Taiwan has maintained zero confirmed cases originating from community spreading for months until the published date of this article. The initial predictions for a high COVID-19 importation risk had turned out to be much lower than expected. The prevalence of other infectious respiratory diseases in Taiwan has also decreased in the meantime as a result of the enumerable disease prevention measures carried out by the CECC through the cooperation of the Taiwanese people.

By coordinating with manufacturers, the number of masks soon became adequate. By adjusting the quota, people can be guaranteed to have masks for self-protection and not live in fear of not having enough masks to use. By allocating the stocks of masks, alcohol for sanitizing, temperature guns, PPEs, and other essential resources, Taiwan reached its adequacy of resources gradually (Ministry of Health and Welfare, 2020f). People and HCWs were not in a panic of insufficiency. Stocks of PPEs helped in Taiwan's disaster preparedness.

The CECC transparently communicated with the general public. People have built a new comprehensive habit to wear a mask almost anywhere and anytime. Following policies of hand washing, hand sanitization, coughing etiquettes, and social distancing has become people's new way of life (Hsih et al., 2020). The Taiwanese people have normalized sanitizing hands and wearing masks before entering public areas such as Mass Rapid Transit, clinics, schools, etc. Most people are now cautious, but not in a panic. This all reflects the success of the CECC's empathetic efforts.

Because of the rapid measures taken to control the epidemic and the efforts from the government to fairly distribute resources to parties in need, hospitals and clinics did not run out of PPEs, and HCWs did not need to panic or complain about the insufficiency of PPEs. Hospitals were not crowded with cases of COVID-19, continuous in-hospital spreading was not observed, and ventilators did not run out. Members in medical facilities are not over-loading. This was all achieved through an organized system of discerning the condition of different patients to determine whether they require medical attention from hospitals or simply need to be confined to quarantine. Because of the near zero domestic infection for months following the peak of the epidemic and how doctors can utilize patient's TOCC history transparently, HCWs could make correct decisions for the need of a SARS-CoV-19 test for a patient.

In conclusion, because the institutions in Taiwan allowed for transparency and a cheap, convenient, and reliable healthcare system, people were able to trust the Taiwanese COVID-19 response team in taking excellent care of them and are thus less afraid of self-reporting a case.

For months without a large-scale outbreak after Taiwan's fleeting epidemic, the government was able to begin to raise economically stimulating policies. People were encouraged to travel within Taiwan, and many scenic spots became thriving pools of consumerism (Taiwan Centers for Disease Control, 2020). With caution, people in Taiwan began to travel, shop, and eat at restaurants for the life of the post-SARS-CoV-2 era.

\section{Challenges and Limitations to the Study}

There are limitations to Taiwan's model as a whole. Given the ephemeral nature of the epidemic, the authors of this study cannot ascertain the applicability of Taiwan's intensive policies in the long run. For example, they cannot vindicate the possibility of the failure of Taiwan's action items toward the end of the COVID-19 pandemic as all policies require efforts in cooperation and acceptance from the public. Thus, designing an epidemic prevention strategy always comes with a risk of depriving large values of funds toward an ineffective set of actions; as such, governments need to modify their models that best suit their people. Additionally, given that most people in Taiwan are not infected with SARS-CoV-2, they do not have protective antibodies to the disease. Until an effective vaccine is invented, a future epidemic of SARS-CoV-2 or its mutated forms may pose 
as yet another arduous endeavor for Taiwan. The risk of a recurring SARS-CoV-2 epidemic in Taiwan is something else that runs within the Taiwan model. One other limitation to the Taiwan model is that Taiwan is a relatively small country compared with others and is thus able to follow up with individual cases more easily compared with other larger countries.

\section{Conclusions}

Taiwan was able to harness the lessons it learned from its 2003 SARS outbreak on the importance of a rapid response to disease outbreaks and strategize to make improvements in preparation for the future. This is evidenced by Taiwan's efforts in setting up laws and establishing SOPs to combat any outbreaks of future respiratory infectious diseases.

Swift reaction, advance deployment, and transparency are the three mainstay elements of Taiwan's epidemic prevention policies. Furthermore, the fulfillment of early and rapid border controls, continued case finding and documentation, the unity of the public through compassion, and resource allocation ensuring the availability of medical supplies collectively led to Taiwan's success.

Taiwan has successfully prevented a large-scale epidemic. Taiwan is the epitome of a citizen-wellness-centered society that understands to respond efficiently and methodologically in times of crises under the world's scrutiny. The Taiwan model works.

\section{References}

Borak, M. (2020, April 17). How a Reddit-like forum helped Taiwan prepare early for Covid-19. Abacus. Retrieved from https://www.scmp.com/abacus/tech/article/3080309/how-reddit-forum-helped-taiwanprepare-early-covid-19

Centers for Disease Control. (2018, December 30). Severe Pneumonia with Novel Pathogens (COVID-19), Nationwide, Indigenous and Imported, Week 1/2019 - Week 31/2020 [Chart]. Severe Pneumonia With Novel Pathogens (COVID-19). https://doi.org/10.1289/isesisee.2018.S01.01.36

Centers for Disease Control and Prevention. (n. d.). [The source details additional COVID-19 cases in Taiwan and is presented by the Taiwan CDC.] [Chart]. COVID-19. Retrieved from https://docs.google.com/ document/d/11XD6o-CbFzCp-4dDVG7SBkWuhdp-KsNPO8C-D-GFmBg/edit?ts=5f166950\#

Department of Disease Control. (2020, May 14). Reserve sufficient high-quality inspection capacity to accurately locate those who may be infected. Department of Disease Control. Retrieved from https://covid19.mohw.gov.tw/ch/cp-4842-53637-205.html

Gardner, L., Zlojutro, A., Rey, D., \& Dong, E. (2020). Modeling the Spread of 2019-nCoV.

Howard, J., Huang, A., Li, Z., Tufekci, Z., Zdimal, V., ... Rimoin, A. W. (2020). Face Masks Against COVID-19: An Evidence Review. Www.Preprints.Org. https://doi.org/10.20944/preprints202004.0203.v1

Hsih, W.-H., Cheng, M.-Y., Ho, M.-W., Chou, C.-H., Lin, P.-C., ... Lu, M.-C. (2020). Featuring COVID-19 cases via screening symptomatic patients with epidemiologic link during flu season in a medical center of central Taiwan. Journal of Microbiology, Immunology, and Infection, 53(3), 459-466. https://doi.org/10.1016/j.jmii.2020.03.008

Jiang, Z. (2020, April 16). Screenshots of PTT broke the news of "Protecting the Country" to help Taiwan grasp first-hand epidemic information. Rti Central Radio. Retrieved from https://www.rti.org.tw/news/view/id/2060071

Lai, C.-C., Shih, T.-P., Ko, W.-C., Tang, H.-J., \& Hsueh, P.-R. (2020). Severe acute respiratory syndrome coronavirus 2 (SARS-CoV-2) and coronavirus disease-2019 (COVID-19): The epidemic and the challenges. International Journal of Antimicrobial Agents, 105924. https://doi.org/10.1016/j.ijantimicag.2020.105924

Lee, P.-C., National Health Insurance Administration, Ministry of Health and Welfare, \& Executive Yuan. (2016, December). National Health Insurance in Taiwan 2016-2017 (bilingual). Retrieved August 2, 2020, from https://www.nhi.gov.tw/Resource/webdata/13767_1_National\%20Health\%20Insurance\%20in\%20Taiwan\% 202016-2017(bilingual).pdf

Ministry of Foreign Affairs. (2020, April 27). VP Chen shares Taiwan's experience combating coronavirus with Johns Hopkins University. Taiwan Today. Retrieved July 31, 2020, from https://taiwantoday.tw/news.php?unit=2,6,10,15,18\&post=176258

Ministry of Health and Welfare. (2019, December 31). COVID-19 Key Decision Timeline for Epidemic Prevention [Chart]. COVID-19 Key Decision Timeline for Epidemic Prevention. Retrieved from 
https://covid19.mohw.gov.tw/ch/sp-timeline0-205.html

Ministry of Health and Welfare. (2019, December 31). Hold a press conference to announce the epidemic information, initiate border quarantine in accordance with standard operating procedures, and board direct flights from Wuhan for quarantine. https://covid19.mohw.gov.tw/ch/cp-4822-53407-205.html

Ministry of Health and Welfare. (2020a, January 5). Determine the case definition and report the case handling process, request to strengthen infection control measures, and strengthen the ability to diagnose emerging infectious diseases. Retrieved from https://covid19.mohw.gov.tw/ch/cp-4822-53413-205.html

Ministry of Health and Welfare. (2020a, March 13). COVID-19 key decision-making network. Retrieved from https://covid19.mohw.gov.tw/ch/cp-4822-53431-205.htm

Ministry of Health and Welfare. (2020b, May 14). Smart community epidemic prevention. Retrieved from https://covid19.mohw.gov.tw/ch/cp-4829-53660-205.html

Ministry of Health and Welfare. (2020c, May 14). Establish border defense lines to stop the virus from overseas [Fact sheet]. Border Policies. Retrieved August 1, 2020, from https://covid19.mohw.gov.tw/ch/cp-4838-536 25-205.html?fbclid=IwAR0KFoPaclQFxceDZKrEbbbmxU7X593wfq4qOF8GXwDY5C8n3rJNDjdrymU

Ministry of Health and Welfare. (2020d, May 14). Open and transparent information. Factors for successful epidemic prevention. $\quad$ Retrieved August 2, 2020, from https://covid19.mohw.gov.tw/ch/cp-4826-53649-205.html

Ministry of Health and Welfare. (2020e, May 29). Stabilize anti-epidemic supplies and stabilize the hearts of the people. Masks and other anti-epidemic materials. Retrieved August 2, 2020, from https://covid19.mohw.gov.tw/ch/cp-4839-53642-205.html

Sui, C. (2020, March 10). What Taiwan can teach the world on fighting the coronavirus. NBC Universal. https://doi.org/10.1155/2020/3703017

Taiwan Centers for Disease Control. (2018, December 17). National Health Command Center (NHCC). About $\begin{array}{lllll}\text { CDC. } & \text { Retrieved } & \text { August } & \text { from }\end{array}$ https:/www.cdc.gov.tw/Category/MPage/6CJ7RCriP1wF4BmtgAhKuA\#

Taiwan Centers for Disease Control. (2018). NHCC.Www.Cdc.Gov.Tw. Retrieved from https://www.cdc.gov.tw/En/Category/MPage/gL7-bARtHyNdrDq882pJ9Q

Taiwan Centers for Disease Control. (2020, July). Policy loosening. Severe special infectious pneumonia. Retrieved August 2, 2020, from https://www.cdc.gov.tw/Category/MPage/doa8zQMXRvr_n3beaD0gSA

Taoyuan International Airport Co., Ltd. (2020, February 14). Personnel quarantine. Immigration. Retrieved August 2, 2020, from https://www.taoyuan-airport.com/main_ch/docdetail.aspx?uid=345\&pid=12\&docid=91\&mc=sys010302

Wang, C. J., Ng, C. Y., \& Brook, R. H. (2020). Response to COVID-19 in Taiwan: Big Data Analytics, New Technology, and Proactive Testing. JAMA. https://doi.org/10.1001/jama.2020.3151

Wang, S., \& Lin, K. (2020, January 6). Foreign visitors to Taiwan up 7\% in 2019 - Focus Taiwan. Retrieved from https://focustaiwan.tw/society/202001060014

World Health Organization. (2020). Coronavirus. $\quad$ Retrieved from https://www.who.int/emergencies/diseases/novel-coronavirus-2019

World Health Organization. (2020, June 29). Timeline of WHO's response to COVID-19. Retrieved from https://www.who.int/news-room/detail/29-06-2020-covidtimeline

\section{Copyrights}

Copyright for this article is retained by the author(s), with first publication rights granted to the journal.

This is an open-access article distributed under the terms and conditions of the Creative Commons Attribution license (http://creativecommons.org/licenses/by/4.0/). 